\title{
Silicon Photonics Technologies for Monolithic Electronic-Photonic Integrated Circuit (EPIC) Applications: Current Progress and Future Outlook
}

\author{
K.-W. Ang, T.-Y. Liow, Q. Fang, M. B. Yu, F. F. Ren, S. Y. Zhu, \\ J. Zhang, J. W. Ng, J. F. Song, Y. Z. Xiong, G. Q. Lo, and D.-L. Kwong \\ Institute of Microelectronics, A*STAR (Agency for Science, Technology and Research), \\ 11 Science Park Road, Singapore Science Park II, Singapore 117685. \\ Phone: +65-6770-5700, Fax:+65-6778-8516, Email: kwongd1@ime.a-star.edu.sg
}

\begin{abstract}
Research into the limits of electrical interconnects indicates that metal wire is unlikely to be the ultimate solution to support the growing functionalities of next generation microprocessor. Severe information latency and power consumption are key technological challenges facing the traditional copper interconnects which impose tremendous constraints to keep up with the performance roadmap known as Moore's Law. The shift of paradigm in computer architecture that enables significant parallelism based on a radically new communication landscape will be a remarkable breakthrough. Converging electronic and photonic integrated circuits (EPIC) on a single chip platform to enable functional diversification emerges as one promising approach which could be realized by taking the advantage of low energy and huge data capacity of optical interconnects. By leveraging on the wealth of CMOS technology know-how and infrastructures, the fundamental photonics building blocks that are essential for the demonstration of low-cost EPIC platform have been successfully developed in this work. We present an overview on the current status of this critical technology development and provide an outlook for the monolithic integration of Si micro- and nano-photonics. A seamless integration of EPIC is poised to become a promising technology to meet the bandwidth and energy requirements of data communication in future technology nodes.
\end{abstract}

\section{INTRODUCTION}

Continuous scaling of complementary metal-oxide-semiconductor (CMOS) transistor technology over the past few decades has led to significant enhancement in the speed performance of microprocessor. As the demand for on-chip functionality continues to grow, it is now widely recognized that electronics technology will increasingly require integration with photonics to keep up with the Moore's Law scaling [1]. Today, high speed data transmission over a bit-rate of $10 \mathrm{~Gb} / \mathrm{s}$ makes photonics interconnects an easier approach to implement than electrical interconnects. Although the exploitation of metal wires with higher conductivity and surrounding dielectrics with lower permittivity had worked out well in the past few generations to minimize signal delay, there is little doubt that such option will offer the ultimate solution. The development of a cost-effective and manufacturable solution that addresses these technological challenges will be a significant breakthrough, such as the optical interconnects technology realized using CMOS-compatible silicon photonics platform.

\section{THE QUEST FOR ELECTRONIC AND PHOTONIC CONVERGENCE AT SYSTEM LEVEL}

The evolution of Moore's Law will soon encounter red-brick walls if the bottleneck of interconnection technology remains unsolved. It is therefore imperative to innovate a revolutionary architecture that could readily support parallelism capable of delivering significant aggregate system performance enhancement, albeit enormous challenges are involved in such implementation. The co-residency of an optical interconnects system and an electronic circuit on a single chip platform is keys to achieving this goal. The exploitation of energy-efficient and high data rate transmission through optical interconnects will be critical to provide high performance functional diversification of CMOS integrated circuits.

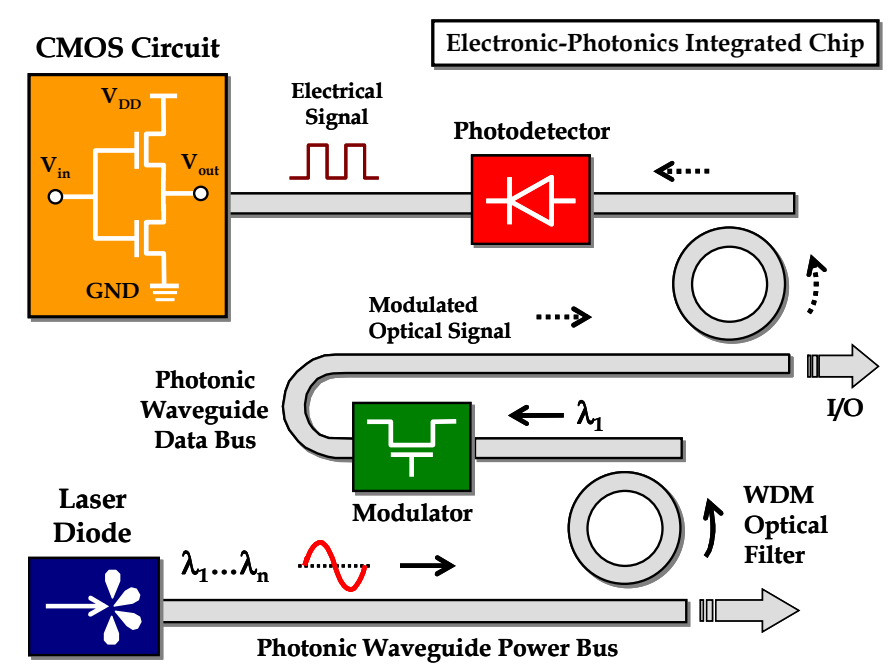

Fig. 1. Schematic illustration of optical interconnects technology realized using Si photonics platform and its convergence with electronics integrated circuit for high performance functional diversification.

Fig. 1 shows a schematic illustration of the essential photonics components needed to construct an optical interconnects system for data transmission and its convergence with CMOS circuit on common $\mathrm{Si}$ platform. In a way analogous to powering the electronic IC using an electrical power supply, a high quantum efficiency laser source can be employed to provide the required optical power in an integrated photonic circuit. By leveraging on the high index contrast between the Si core and the silicon-dioxide cladding, routing of optical signals using photonic micro-waveguide with low propagation loss can be made possible. Furthermore, to harness increased interconnects capacity, a wavelength division multiplexing (WDM) could be exploited to provide multiple channels transmission for parallel data processing. Using an optical filter made of ring resonator or arrayed waveguide grating (AWG), a selected wavelength $(\lambda n)$ can be filtered into the photonic waveguide data bus. While Si-based modulator exhibits weaker electro-optical coefficients due to its central symmetry in the lattice structure, a free carrier plasma dispersion effect can be exploited to enable effective optical modulation. The modulated optical signals are then channeled through the photonic waveguide data bus where multiple wavelengths signals can be delivered on the same channel without suffering interference from one another. At the receiving end, signal of a specific wavelength channel is filtered and fed into a high performance photodetector for enabling efficient opticalto-electrical encoding. The encoded electrical signal will then pass through the CMOS logic circuitry for data processing. Needless to say, the development of both active and passive photonics building blocks will be crucial to bring EPIC convergence into reality. 


\section{ENABLING TECHNOLOGY AND FUNDAMENTAL BUILDING BLOCKS DEVELOPMENT}

\section{A. High Performance Ge-Based Photodetectors (PD) \\ Advanced Low-Temperature Selective Germanium Epitaxy}

Recent advancement shows that germanium $(\mathrm{Ge})$ is attracting growing interest to enable the realization of highly efficient photodetector (PD) for its favorable absorption coefficient in the near-infrared wavelengths regime. However, technological challenges exist for the epitaxy growth of high quality germanium $(\mathrm{Ge})$ film on silicon $(\mathrm{Si})$ due to the large lattice mismatch between the two heterostructure materials. The existence of $\sim 4.2 \%$ lattice strain has been shown to give rise to two major issues: (1) high densities of threading dislocations and (2) rough surface morphology due to 3D Stranski-Krastanov (SK) growth. Both of these defects present much concerns for the generation of high leakage current which would compromise the efficiency of a photodetector. Unlike the conventional approaches of employing thick SiGe buffer layer, we have developed a low temperature pseudo-graded SiGe buffer engineering approach to relieve the large lattice mismatch stress [2], thereby suppresses the formation of dislocation defects substantially (Fig. 2). This allows the elimination of high temperature post-epitaxy anneal that is commonly used for defects reduction, which renders it attractive for CMOS implementation due to the introduction of a low thermal budget $\left(<700^{\circ} \mathrm{C}\right)$.

\section{Germanium-Photodetector and Its Portfolio}

Fig. 3 shows the photodetector portfolio developed in this work, which include waveguided and normal incidence Ge PIN PD, Ge MSM $\mathrm{PD}$ as well as $\mathrm{Ge} / \mathrm{Si}$ heterostructure avalanche PD [2]-[10]. The recent technological breakthrough in the demonstration of Ge/Si APD with high gain-bandwidth product deserves a special mention here [3]. Due to an internal carrier multiplication mechanism via avalanche effect, a very high responsivity performance of $>3.3 \mathrm{~A} / \mathrm{W}$ and a low dark current density of $\sim 3 \mathrm{nA} / \mu \mathrm{m}^{2}$ were achieved at an operating voltage of $90 \% \mathrm{~V}_{\mathrm{b}}$ (Fig. 4). This gives rise to a sensitivity of $-27.5 \mathrm{dBm}$ at a bit-rate of 10 $\mathrm{Gb} / \mathrm{s}$, showing comparable performance to that of III-V based APD. Moreover, a CMOS TIA packaged Ge/Si APD achieved a very impressive gain-bandwidth product of $\sim 180 \mathrm{GHz}$. In addition, by leveraging on our in-house packaging capability, a single-mode lensed fiber pig-tail (SFP) optical receiver featuring waveguided Ge PIN PD has also been demonstrated with a bit rate performance of $10 \mathrm{~Gb} / \mathrm{s}$, as shown by the eye pattern (PRBS $2^{7}-1$ ) measurement (Fig. 5).

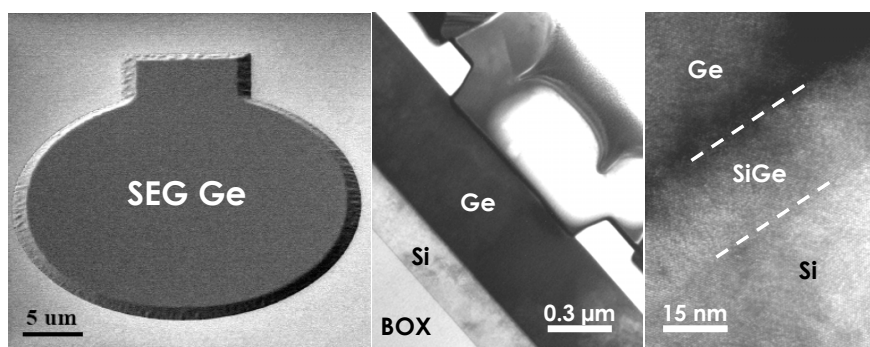

Fig. 2. The achievement of high quality selective Ge epitaxy growth via low temperature pseudo-graded SiGe buffer engineering.

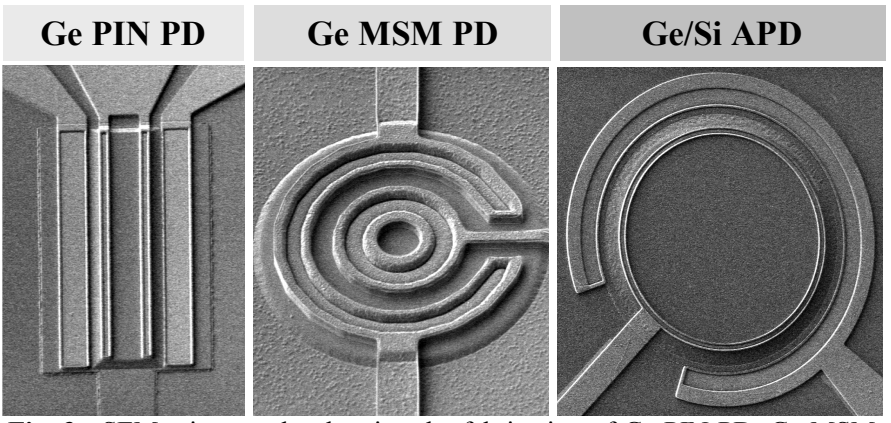

Fig. 3. SEM micrographs showing the fabrication of Ge PIN PD, Ge MSM $\mathrm{PD}$, and $\mathrm{Ge} / \mathrm{Si} \mathrm{APD}$ using high quality selective Ge epitaxy process.

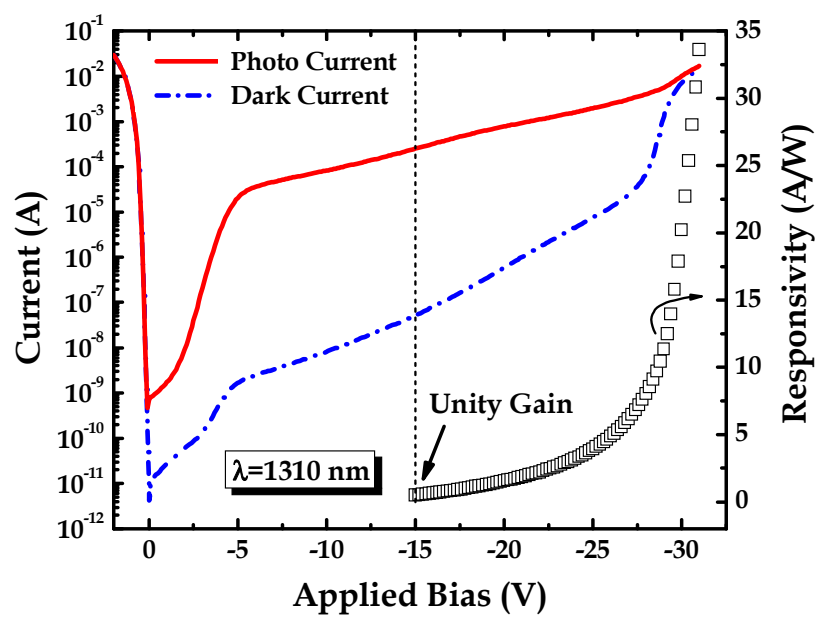

Fig. 4. Due to an internal multiplication gain, very impressive responsivity performance was measured in a Ge/Si APD at a wavelength of $1310 \mathrm{~nm}$.

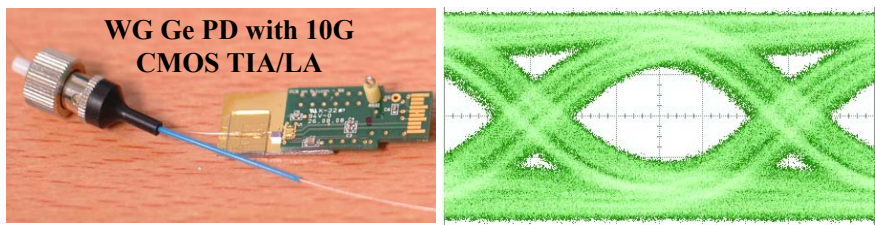

Fig. 5. A packaged optical receiver featuring waveguide Ge PD and CMOS TIA/LA circuit. An eye pattern (PRBS $2^{7}-1$ ) of $10 \mathrm{~Gb} / \mathrm{s}$ was demonstrated.

\section{B. Low Power and High Speed Si Modulators (MOD)}

Modulators can be monolithically fabricated on silicon by exploiting the free carrier plasma dispersion effect [11]. For wideband operation across a range of wavelengths, the Mach-Zehnder design is typically utilized. By inserting $p$ - $n$ junction phase-shifters in the Si waveguides, the carrier density can be controlled to enable optical intensity modulation at the output. Operation of the phase-shifters in depletion mode results in excellent modulator speed characteristics [12], Intel has demonstrated modulation speeds of up to $40 \mathrm{Gbps}$ in carrier depletion type modulators with 1-mm-long phase shifters. However, a relatively low extinction ratio (E.R.) of $1.1 \mathrm{~dB}$ was reported. For $10 \mathrm{Gbps}$ operation, they reported an extinction ratio (E.R.) of $5.2 \mathrm{~dB}$ for a modulator with 3-mm-long phase-shifters, which were differentially driven with RF signals of $7.6 \mathrm{Vpp}$ each. Here, we report a carrier depletion type modulator with comparable if not better phase-shifting efficiency $\left(V_{\pi} L_{\pi} \sim 2.56 \mathrm{Vcm}\right)$ compared with the best reported carrier depletion type modulators [12]-[16]. For 10 Gbps operation, an E.R. of $6.1 \mathrm{~dB}$ was achieved for a modulator with a 2-mm-long phase-shifter, which was single-endedly driven with a RF signal of $5 \mathrm{Vpp}$. The free carrier density in the rib waveguide phase-shifters is modulated by varying the depletion width of the $p$ - $n$ junction.

The free carrier density in the rib waveguide phase-shifters is modulated by varying the depletion width of the $p-n$ junction. As free holes are more effective in perturbing the index than free electrons, interaction between the optical mode and the regions of hole density change should be maximized by tightly confining the optical mode within the rib waveguide, and by controlling the dopant profile and the location of the $p$ - $n$ junction. Fig. 6 plots the extracted phase-shift $\Delta \varphi$ and normalized capacitance at different phase-shifter voltages [17]. Phaseshifting is more efficient in the low voltage regime between $-1 \mathrm{~V}$ to +0.7 $\mathrm{V}$, as can be expected from the depletion characteristics of a $p-n$ junction. However, there is a trade-off between efficiency and speed, since the normalized capacitance at lower reverse voltages is also higher due to the smaller depletion width. Depending on the requirements, the operating point of the modulator can be set using the DC bias. 


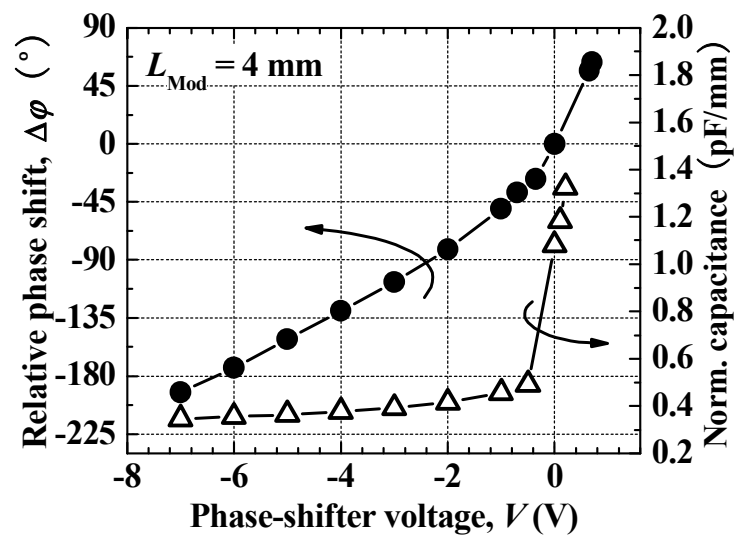

Fig. 6. Relative phase-shift and normalized capacitance plotted against phase-shifter voltages. The phase-shifting efficiency is higher for phaseshifter voltages from $-1 \mathrm{~V}$ to $+0.7 \mathrm{~V}$. However, the normalized capacitance is also higher due to the smaller depletion width of the $p$ - $n$ junction.

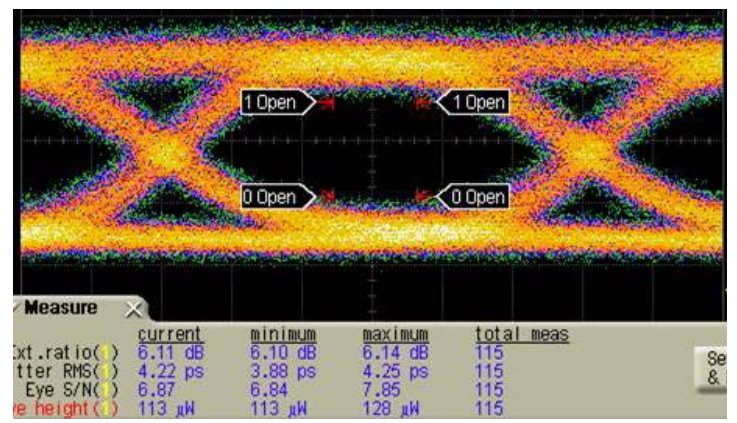

Fig. 7. Eye pattern measurements for modulator with 2-mm-long phaseshifter driven at $10 \mathrm{Gbps}$ in single-ended mode $\left(V_{\mathrm{DC}}=-3.75 \mathrm{~V}, V_{\mathrm{RF}}=5 \mathrm{~V}_{\mathrm{pp}}\right)$. The measured E.R. and jitter is about $6.1 \mathrm{~dB}$ and $\sim 4.2$ ps.

A modulator with a 2-mm-long phase-shifter was driven in singleended mode at $10 \mathrm{Gbps}$ (only one arm is driven, $V_{\mathrm{DC}}=-3.75 \mathrm{~V}, V_{\mathrm{RF}}=5$ $\mathrm{V}_{\mathrm{pp}}$ ). The chosen $V_{\mathrm{DC}}$ ensures operation in the low capacitance regime. An E.R. of $\sim 6.1 \mathrm{~dB}$ and jitter of $\sim 4.2$ ps was measured at 10 Gbps [Fig. 7], which is the speed limit of the measurement equipment used. The short rise/fall times indicate that even higher speed is possible. At the other extreme, low voltage operation $\left(V_{\mathrm{RF}}=1 \mathrm{~V}_{\mathrm{pp}}, V_{\mathrm{DC}}=0 \mathrm{~V}\right)$ was also demonstrated in differentially-driven modulators with 4-mm-long phase shifters, at the expense of speed (3.125 Gbps).

\section{Si-Based Photonic Crystal Optical Source}

The realization of a Si-compatible light source has been known as one of the "holy grails" for enabling monolithic integration of electronic and photonic integrated circuits. For successful EPIC implementation, a high performance laser which meets the following criteria will be desirable: (1) it allows efficient emission at standard communication wavelength of $1550 \mathrm{~nm}$, (2) it is fully compatible with current CMOS fabrication technology, and (3) it can be electrically pumped. Conventional approaches adopted to fulfill these criteria include the use of hybrid bonded III-V compound laser on $\mathrm{Si}$, erbium-doped $\mathrm{Si}$ rich oxide or nitride, and $\mathrm{Si} / \mathrm{SiGe}$ nanocrystal. However, none of the above approach is capable of meeting all these three requirements thus far.

In this work, a 3D-confined structure with triangular-lattice air-hole photonic crystal patterns was fabricated to enhance the light output from silicon rich oxide $/ \mathrm{SiO}_{2}$ multiple quantum well (Fig. 8) [18]. The adoption of PC enhances the external quantum efficiency significantly, thereby serves to increase the light output efficiency. The mechanisms for such enhancement are attributed to the coherent scattering of the internal trapped light and the quantum electrodynamic effects which resulted in a concentration of EM fields in the active material (Fig. 9).
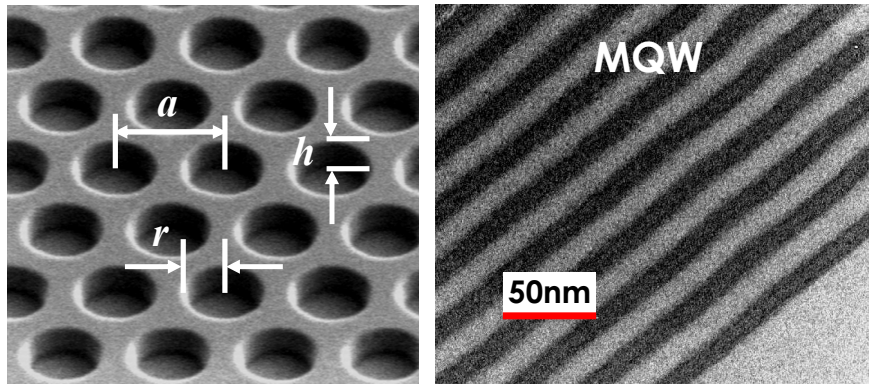

Fig. 8. (a) SEM top view of a $2 \mathrm{D}$ triangular-lattice air-hole photonic crystal (PC) geometry. (b) X-TEM micrograph of the active region composed of ten periods of $\mathrm{SRO} / \mathrm{SiO}_{2} \mathrm{MQW}$.

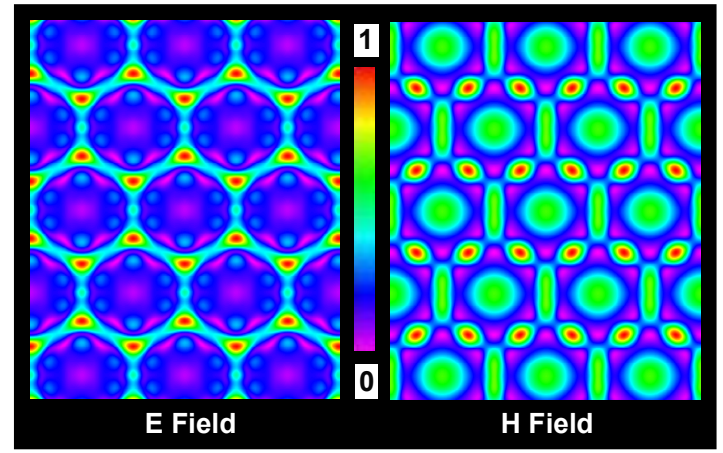

Fig. 9. The normalized (a) electric field intensity distribution and (b) magnetic field intensity distribution of excitation incidence $(325 \mathrm{~nm})$ in the plane of $h / 2$ for the sample with pattern E [18].

In yet another approach, we have investigated the light emission using amorphous $\mathrm{Si} / \mathrm{Si}_{3} \mathrm{~N}_{4}$ multiple-quantum-well structures, in which twodimensional hexagonal-lattice air-hole photonic crystals (PCs) were integrated [19]. Significant light extraction enhancement was achieved due to strong coupling of the inherent leaky modes or radiation modes near Gamma point of the PC's band structure. Recent progresses made in our group have even shown that with erbium (Er) doping in the PC arrays, near-infrared wavelength emission $(\sim 1.5 \mu \mathrm{m})$ was made possible.

\section{Passive Photonics Components}

\section{Passive Device Library based on High-Index-Contrasting System}

Passive photonics components form an important integral for EPIC implementation. Passive device library developed in this work includes nano-tip, filter, directional coupler, splitter, ring resonator, arrayed waveguide grating $(A W G)$ and most importantly SOI waveguides for routing optical signals on the photonics integrated circuit [20]-[25]. With the availability of a high-index-contrast (HIC) system (e.g. Si as core waveguide and $\mathrm{SiO}_{2}$ as cladding), miniaturization of photonic circuit becomes feasible due to the possibility of reducing the bend radius to $<5 \mu \mathrm{m}$. However, the adverse consequence of HIC lies with the increased sensitivity of sidewall scattering which affects the optical propagation loss. Using state-of-the-art CMOS process technology, negligible waveguide transmission loss of $\sim 0.5 \mathrm{~dB} / \mathrm{cm}$ has been demonstrated in this work.

Wavelength-Division-Multiplexing and Array-Waveguide-Grating

Fig. 10 shows the wavelength division multiplexing (WDM) based on (a) 32-channels AWG composed of rib SOI waveguides and (b) ring resonator in photonic domain to provide multiple channels for parallel data processing without signal interference. The adoption of WDM offers the capability to enable simultaneous multi-channel transmission to scale the aggregate data-rate beyond the limit of existing electrical interconnect scheme. 

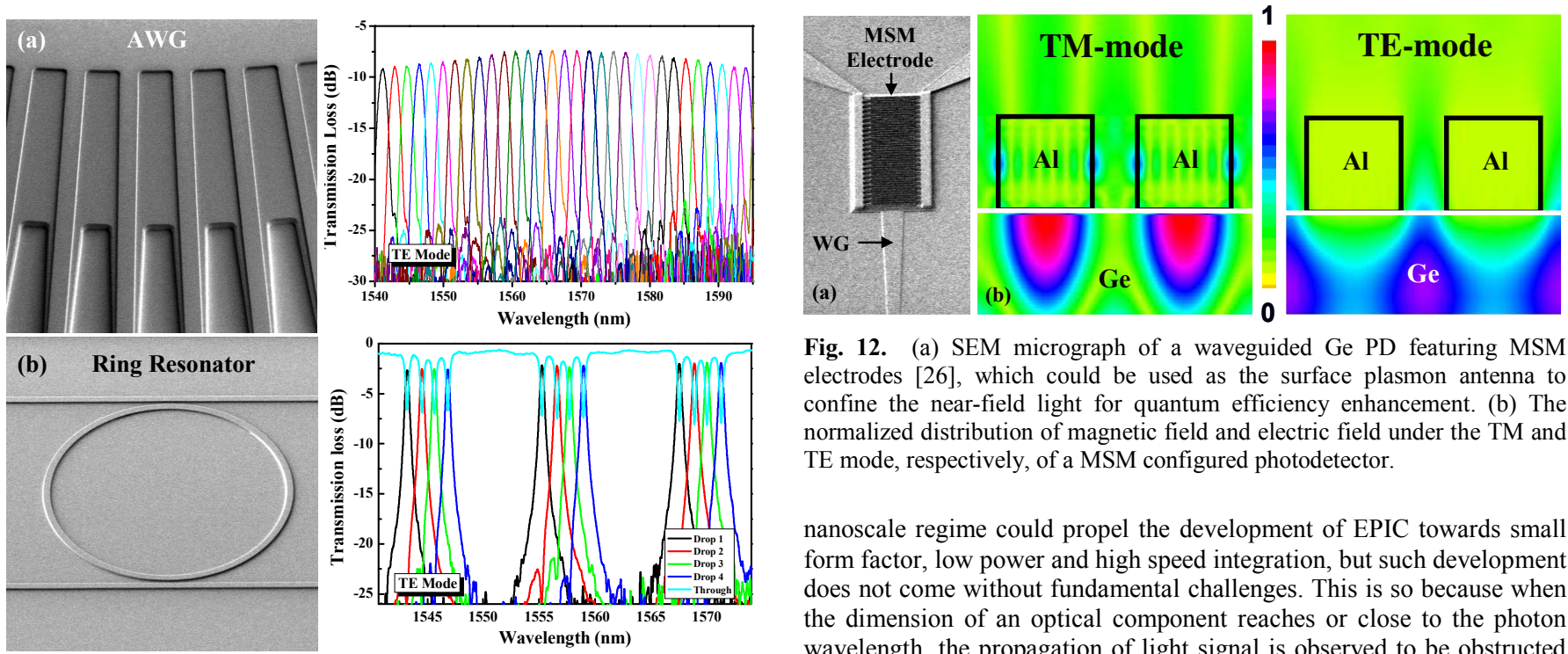

Fig. 12. (a) SEM micrograph of a waveguided Ge PD featuring MSM electrodes [26], which could be used as the surface plasmon antenna to confine the near-field light for quantum efficiency enhancement. (b) The normalized distribution of magnetic field and electric field under the TM and TE mode, respectively, of a MSM configured photodetector.

nanoscale regime could propel the development of EPIC towards small form factor, low power and high speed integration, but such development does not come without fundamental challenges. This is so because when the dimension of an optical component reaches or close to the photon wavelength, the propagation of light signal is observed to be obstructed by the optical diffraction limit. The exploitation of surface plasmon (SP) based nanophotonics may offer a solution to alleviate this problem as the feature of SP enables the possibility of localization and guiding of light in sub-wavelength structures. Fig. 12 shows recent demonstration of a surface plasmon antenna technology to boost the quantum efficiency of a miniaturized photodetector [26]. In this device, interdigitated metallic electrodes acting as one-dimensional rectangular gratings are employed to enhance the TM-mode responsivity, which typically exhibits weaker response than the TE-mode response. Such remarkable enhancement is attributed to the reduced group velocity at the photonic band edge of TM mode, which concomitantly leads to a strong optical confinement and resonance within the Ge region. This in turn enables effective absorption. As projected, the application of plasmonics would enable the next wave of research and development in nanophotonics to realize ultra low power and high data rate optical interconnect solutions in the nanoscale regime.

\section{CONCLUSION}

fabrication feasibility. In such monolithic integration scheme, an 'electronic-first and photonic-last' integration approach was proposed and demonstrated [2]. In this approach, all high temperature processes $\left(>1000^{\circ} \mathrm{C}\right)$ needed to form CMOS logic circuit are performed first prior to the fabrication of photonic components and circuit. Fig. 11 shows (a) the PD-SOI CMOS inverter circuit and (b) the integrated Si modulator and Ge photodetector realized using the monolithic technology platform developed in this work.

Ultimately, what limits the integration of optical and electronic circuits most is the mismatch in their respective length scales. This is true as electronics devices continue to scale in advanced CMOS technology nodes, the foot-print of existing photonics devices which are typically in the micrometer scale seems to impose much constraint to enable the realization of ultra-compact electronic-photonic integrated circuit (EPIC). Aggressive miniaturization of photonic devices into the
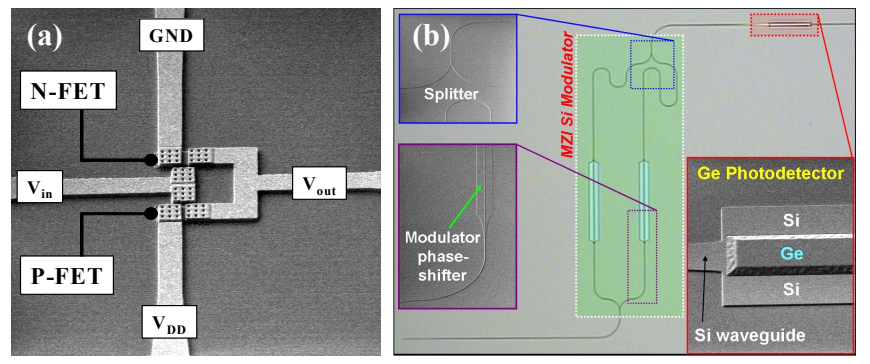

Fig. 11. (a) SEM micrograph of a Si CMOS inverter circuit integrated on SOI platform. Partially depleted CMOS transistors were fabricated [7]. (b) Monolithically integrated Si optical modulator and Ge photodetector [22].

The recent progress of silicon photonics technology development for enabling EPIC convergence to meet the growing functionality of next generation microprocessor has been reviewed. Photonics interconnects show great potential to enable data capacity scalability while consuming less power. Key competitive enabling technologies have been developed, along with a set of well established active and passive photonics device libraries. It is believed that nanophotonics technologies can potentially change the landscape for Silicon Photonics, allowing for greater miniaturization and taking the performance to new heights.

\section{REFERENCES}

[1] L. C. Kimerling et al., Proc.SPIE, 6125, 12502, 2006. [2] K.-W. Ang et al, IEEE J. Sel. Topics Quantum Elect., 14, 2010. [3] X. Wang et al, The Opt. Fiber Comm. Conf., 2009. [4] J. Wang et al, IEEE Photon. Tech. Lett., 20, 1485, 2008 [5] S. Y. Zhu et al, IEEE Photon. Tech. Lett., 20, 1396, 2008. [6] K.-W. Ang et al, IEEE Elect. Dev. Lett., 29, 704, 2008. [7] K.-W. Ang et al, IEEE Photon. Tech. Lett., 20, 754, 2008. [8] K.-W. Ang et al, IEEE Elect. Dev. Lett., 29, 708, 2008. [9] H. Zhang et al, IEEE Elect. Dev. Lett., 29, 161, 2008. [10] T. H. Loh et al., Appl. Phys. Lett., 91, 073503, 2007. [11] R. Soref et al., IEEE J. Quantum Elect., 23, 123, 1987. [12] F.Y. Gardes et al, Opt. Express, 13, 8845, 2006. [13] T. Pinguet et al., Proc. SPIE, 6898, 689805, 2008. [14] D. Marris-Morini et al., Opt. Express, 16, 334, 2008. [15] A. Liu et al., Opt. Express, 15, 660, 2007. [16] Q. Xu et al., Opt. Express, 15, 430, 2007. [17] T.-Y. Liow et al, IEEE J. Sel. Topics Quantum Elect., 14, 2010. [18] F. F. Ren et al., Appl. Phys. Lett., 93, 091901, 2008. [19] F. F. Ren et al., IEEE Photon. Tech. Lett., 21, 91, 2009. [20] Q. Fang et al, IEEE Photon. Tech. Lett., 21, 319, 2009. [21] J. F. Song et al., Opt. Express, 16, 21476, 2008. [22] S. H. Tao et al., Opt. Express, 16, 21456, 2008. [23] S. C. Mao et al., Opt. Express, 16, 20809, 2008. [24] J. F. Song et al., Opt. Express, 16, 15304, 2008. [25] Q. Fang et al., Opt. Express, 16, 6425, 2008. [26] F. F. Ren et al., submitted to Appl. Phys. Lett., 2009. 